\title{
El procesamiento de oraciones y la interfaz sintaxis-semántica: contraste entre dos modelos psicolingüísticos
}

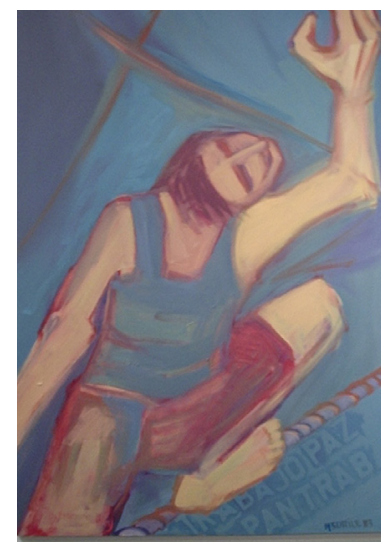

Carolina A. Gattei

Instituto de Física de Buenos Aires, CONICET; Laboratorio de Neurociencia, UTDT, Argentina

carolina.gattei@conicet.gov.ar

\section{Luis A. París}

Grupo de Lingüística y Neurobiología del Lenguaje, Instituto de Ciencias Humanas, Sociales y Ambientales, CONICET, Argentina

paris@mendoza-conicet.gov.ar

Trabajo recibido el 12 de abril de 2018 y aprobado el 27 de septiembre de 2018.

\section{Resumen}

El presente trabajo describe los modelos psicolingüísticos relevantes para explicar cómo se lleva a cabo el enlace entre sintaxis y semántica durante el procesamiento de oraciones en tiempo real. La realización de dicho enlace permite a los hablantes interpretar 'quién hace qué a quién' de manera correcta. Se presentan dos modelos que tienen en cuenta las diferencias específicas de cada lengua, a la vez que proponen un mecanismo universal para la interpretación de cláusulas simples: el Extended Argument Dependency Model y el Linking and Checking Model. Estos modelos pretenden explicar cómo se llevarían a cabo estos procesos durante la comprensión incremental desde dos marcos teóricos de la lingüística formal: la Gramática del Rol y la Referencia y el Generativismo. Luego se expone cómo resolverían dichos modelos la interpretación de un evento cuyo enlace entre sintaxis y semántica es no canónico y se discute qué modelo tiene mayor poder explicativo.

Palabras clave

interfaz sintaxis-semántica procesamiento de oraciones modelos psicolingüísticos prominencia

\section{The processing of sentences and the syntax-semantics interface: Contrast between two psycholinguistic models}

\begin{abstract}
The current work describes the relevant psycholinguistic models that explain how the link between syntax and semantics is carried out during the processing of sentences in real time. The implementation of this link allows speakers
\end{abstract}

\section{Keywords}

syntax-semantics interface processing of sentences psycholinguistic models prominence 
to interpret 'who does what to whom' correctly. Two models are presented which take into account the specific differences of each language and at the same time they propose a universal mechanism for the interpretation of simple clauses: the Extended Argument Dependency Model and the Linking and Checking Model. These models try to explain how these processes would be carried out during the incremental comprehension from two theoretical frameworks of formal linguistics: the Role and Reference Grammar and Generativism. Then it is presented how these models would resolve the interpretation of an event whose link between syntax and semantics is noncanonical and it is discussed which model has a greater explanatory power.

\section{O processamento de sentenças e a interface sintaxe-semântica: Contraste entre dois modelos psicolinguísticos}

\section{Resumo}

Este trabalho descreve os modelos psicolinguísticos relevantes para explicar como é levado a cabo o enlace entre sintaxe e semântica durante o processamento de sentenças em tempo real. A realização desse enlace permite aos falantes interpretarem 'quem faz o quê a quem' corretamente. São apresentados dois modelos, que levam em consideração as diferenças específicas de cada língua, e ao mesmo tempo propõem um mecanismo universal para a interpretação de cláusulas simples: o Extended Argument Dependency Model e o Linking and Checking Model. Estes modelos procuram explicar como esses processos poderiam ser levados a cabo durante a compreensão incremental a partir de dois marcos teóricos da linguística formal: a Gramática de Papel e Referência e o Gerativismo. Depois, se expõe como esses modelos resolveriam a interpretação de um evento cujo enlace entre sintaxe e semântica é não canônico e se discute o que modelo tem o maior poder explicativo.

\section{Introducción}

El procesamiento de oraciones - tanto producción como comprensióninvolucra la integración sistemática de distintos tipos de información y, consecuentemente, los fenómenos de interfaz (más precisamente, el enlace entre los niveles de información sintáctica y semántica) han generado reciente interés en el campo de la psico y la neurolingüística. Su estudio permite comprender cómo la interacción de distintos tipos de información lingüística afecta la interpretación de eventos. Esto es de crucial importancia para cualquier modelo que procure explicar cuáles son los mecanismos subyacentes a la comprensión de lenguaje, ya que el ser humano sólo puede comprender eventos - es decir, quién hizo qué a quién-a través de la correcta asociación entre información sintáctica y semántica.

La evidencia psicolingüística sugiere que el procesador de lenguaje funciona de manera incremental, es decir que no espera hasta tener una representación lingüística acabada de un enunciado para construir una representación sintáctica, sino que construye una secuencia de representaciones parciales que se actualiza con cada pieza de información lingüística entrante (Tanenhaus et al. 1995, Konieczny 2000, Kamide y Mitchell 1999). Estas representaciones involucran una anticipación, es decir, no son sólo sobre el
Palavras-chave

interface sintaxe-semântica processamento de sentenças modelos psicolinguísticos proeminência 
estímulo percibido en un momento determinado - una palabra - sino sobre toda la frase y la oración. En otras palabras, el procesamiento incremental aduce que los sujetos predicen la representación del todo a partir de datos incompletos, incluso sumamente escuetos.

Varios modelos han intentado explicar cómo se realiza el procesamiento incremental de lenguaje. Los primeros modelos han basado gran parte de su constructo en evidencia proveniente del procesamiento de oraciones sintácticamente ambiguas en las que se daba un efecto conocido como garden path (en español, efecto de vía muerta). Este efecto consiste en asignar una interpretación sintáctica determinada a la oración y comprobar, con la actualización de la información nueva entrante, que la estructura sintáctica es diferente y la interpretación asignada, incorrecta (Frazier y Fodor 1978, Ferreira y Henderson 1991, Kimball 1975, Altmann y Kamide 1999, Arai y Keller 2013). Kimball (1975) ha observado también que los seres humanos utilizan mecanismos de predicción para posibilitar la comprensión en tiempo real de manera eficiente. La pregunta que surgió inmediatamente es cuál es exactamente el alcance del carácter predictivo del procesador del lenguaje. No sólo se realizan predicciones acerca del tipo de estructura sintáctica que tendrá lugar en la oración (como en el caso de las oraciones que producen el efecto de vía muerta, ver DeLong, Quante, y Kutas 2014; Brown y Hagoort 1993, Kutas, DeLong y Smith 2011; Kutas y Hillyard 1983), sino también sobre la información léxica y de conocimiento de mundo (Chwilla y Kolk 2005, Hagoort et al. 2004, Metzner et al. 2015). Estos estudios muestran una facilitación en la comprensión si las predicciones se verifican en contraste con oraciones donde no se puede predecir o donde las predicciones resultan incorrectas.

Una incipiente pero creciente cantidad de evidencia proveniente del estudio del alemán, una lengua que permite estructuras con verbo final, ha mostrado que los mecanismos de predicción involucran el sistema de enlace sintaxis-semántica (Bader y Bayer 2006; Bornkessel y Schlesewsky 2006; Bornkessel et al. 2005; Bornkessel, Schlesewsky y Friederici 2003). En estas estructuras, la interpretación sobre quién hizo qué a quién sólo es revelada con la aparición del verbo al final de la cláusula, como se observa en (1):

(1) Maria $\quad$ glaubt...
María creer $_{[3 r a-S G]} \ldots$
'María cree...'

(a) ... dass der Priester $\quad$ dem Gärtner folgt.
... que $\mathrm{el}_{[\mathrm{NOM}]}$ cura $\mathrm{el}_{[\mathrm{DAT}]}$ jardinero seguir $_{[\text {[ra-SG] }}$
'... que el cura sigue al jardinero'.

(b) ... dass der Priester $\quad$ dem Gärtner imponiert.
... que $\mathrm{el}_{[\mathrm{NOM}]}$ cura $\mathrm{el}_{[\mathrm{DAT}]}$ jardinero impresionar ${ }_{[\mathrm{BA}-\mathrm{SG}]}$
'... que al jardinero le impresiona el cura'.

(Bornkessel, Schlesewsky y Friederici 2003)

En estos ejemplos, es sólo hacia el final de la oración que los lectores pueden efectivamente comprender que en una oración como (1a) el cura es quien realiza una acción (i.e., es el Actor) y en una oración como (1b) es la persona que despierta un sentimiento en el jardinero (i.e., es el 'Undergoer', Van Valin 2005). El verbo acarrea información semántica 
crucial que selecciona un tipo de enlace, es decir, qué función sintáctica - sujeto, objeto directo e indirecto- se conecta con qué función semántica -'Actor', 'Experimentante', 'Paciente'-. Los estudios gramaticales han explicitado el sistema que gobierna el enlace y lo han vinculado a la semántica verbal.

La evidencia sobre procesamiento de oraciones con diferente complejidad argumental recolectada en los últimos años ha conducido a la postulación de unos pocos modelos psicolingüísticos que han incorporado la noción de enlace o linking dentro de sus supuestos generales. Dos de los modelos más relevantes son el Extended Argument Dependency Model (eADM: Bornkessel y Schlesewsky 2006, Bornkessel-Schlesewsky y Schlesewsky 2008, 2009) y el Linking and Checking Model (LCM: Bader y Bayer 2006). Este trabajo se propone describir las características generales, los supuestos y predicciones de estos modelos y postular su relevancia para el campo de la psicolingüística. Por último, se exponen las diferencias teóricas entre ambos modelos que permiten optar de manera crítica por aquel que posee mayor poder explicativo para dar cuenta de los fenómenos lingüísticos provenientes del nivel de interfaz sintaxis-semántica.

\section{Extended Argument Dependency Model (eADM)}

El Extended Argument Dependency Model (eADM) es un modelo neurocognitivo de comprensión de oraciones que tiene como objetivo principal describir y explicar los correlatos neurofisiológicos, neuroanatómicos y cognitivos de la comprensión de estructuras sintácticas simples (i.e., el verbo y sus argumentos). Una de las motivaciones más relevantes para la postulación de este modelo es el análisis de la variación e, incluso, la contradicción que aparece en la evidencia cuando se evalúa el mismo tipo de estructuras en distintas lenguas. Es, por tanto, un modelo interlingüístico, y sus supuestos teóricos formales están alineados con lo propuesto por la Gramática del Rol y la Referencia (RRG por sus siglas en inglés: Foley y Van Valin Jr. 1984, Van Valin Jr. 2005, Van Valin y LaPolla 1997) tanto en lo que respecta a la concepción de la estructura sintáctica de las oraciones que forman parte del lenguaje (una estructura compuesta por un centro - core - del que se desprenden el núcleo verbal y los argumentos) como a la noción de que existe una representación léxico-semántica que se asocia con la información argumental a través de un algoritmo de enlace. El modelo intenta, entonces, explicar cómo interactúan los distintos tipos de información lingüística para la comprensión de oraciones en línea.

El modelo basa sus supuestos y predicciones en evidencia neurocognitiva interlingüística sobre cómo lidia el procesador de lenguaje con oraciones con distinta complejidad argumental. Esta complejidad está dada por la variación de distintos tipos de información sintáctico-semántica que afectan la jerarquización argumental, tales como el orden oracional, la variación en tipo de verbo, diferencias en la marca de caso, la animacidad y la definitud.

\subsection{Estructura del modelo}

En cuanto a su estructura, el eADM toma algunas características del modelo Neurocognitivo de Comprensión de Oraciones (Friederici 1998, Friederici 2002), un modelo de procesamiento de tres fases con dependencias 
jerárquicas entre cada fase. Sin embargo, el eADM difiere del modelo de Friederici en cuanto a los procesos llevados a cabo en cada una de las fases y a la manera de analizar la información de entrada (ver Bornkessel y Schlesewsky 2006 para una discusión sobre el tema).

\subsubsection{Fase 1}

En una primera fase, el sistema de procesamiento de lenguaje $o$ parser analiza la información formal más básica del input (i.e., información sobre clase de palabra). La evidencia para postular este tipo de fase proviene de estudios en los que se utilizaron violaciones de categoría de palabra (del tipo Max's of proof the theorem), y en los que se encontró una negatividad temprana con topografía anterior izquierda (o ELAN, por sus siglas en inglés para Early Left Anterior Negativity) con la aparición de la palabra of (Hahne y Friederici 1999, Neville et al. 1991, Osterhout y Holcomb 1992, 1993). Por otro lado, el procesamiento de categorías de palabras que no son las predichas pero que de todas maneras pueden integrarse a la estructura sintáctica propuesta, no engendran un ELAN (Friederici 2002). En eADM, esta diferenciación entre lo que es imposible integrar y lo que puede hacerse se explica mediante la noción de plantillas sintácticas, que son estructuras de frase precompiladas que solamente codifican información sobre categoría de palabra. Desde este punto de vista, el ELAN refleja una falla en la selección de la plantilla, que ocurre cuando el inventario de plantillas de la lengua que está siendo procesada no contiene una plantilla compatible con la información que está entrando al sistema.

\subsubsection{Fase 2}

El foco del modelo está puesto en la segunda fase de procesamiento. Esta fase involucra los procesos relacionales que aplican a los argumentos y a los verbos. El modelo propone dos etapas en esta segunda fase: una etapa de activación (fase 2a) y una etapa de computación (fase 2b). A continuación se describe en qué consiste cada una de ellas y en qué difiere el procesamiento de argumentos y verbos.

\subsubsection{Procesamiento de argumentos}

Como puede verse en la figura 1, el modelo asume que el procesamiento de argumentos en la fase 2 es dual: por un lado, una de las tareas del sistema de procesamiento en esta fase es computar el estatus de prominencia para el argumento bajo consideración. La prominencia es la información utilizada para construir una jerarquía interpretativa entre los argumentos de una oración y, de acuerdo con los autores, la asignación del estatus de prominencia está basada tanto en información morfosintáctica (caso morfológico y posición del argumento) como en un pequeño conjunto de tipos de información semántica motivado interlingüísticamente y estructurado de manera jerárquica (e.g., animacidad y definitud de los argumentos). Por otro lado, el sistema de procesamiento asigna al argumento propiedades de concordancia mediante el rasgo de concordancia ([+/-agrt] de acuerdo con la terminología en inglés). Estos dos procesos equivalen a un análisis argumental en términos interpretativos (prominencia) y formal (concordancia), respectivamente. El modelo postula que la prominencia y la 
concordancia están interconectadas siempre que haya información suficiente para la asignación del estatus de prominencia. Por ejemplo, el reconocimiento del tipo de caso de un argumento lleva a la asignación de los rasgos [+agrt] o [-agrt] conforme se trate de caso nominativo o no. Cuando la prominencia no puede ser computada por falta de información, la concordancia es asignada mediante el principio de 'Minimalidad'. Este principio está basado en la idea de que el procesador asume la estructura sintáctica y la interpretación más simple compatible con los elementos que ya han aparecido. A continuación se discuten las manifestaciones neurofisiológicas relacionadas con la etapa computar prominencia, que es la etapa de mayor relevancia para los fenómenos relacionados con la predicción del enlace entre sintaxis y semántica.

\section{Fase 1}

Fase 2

\begin{tabular}{|ll|}
\hline $2 a$ & $2 b$ \\
\hline
\end{tabular}

Fase 3

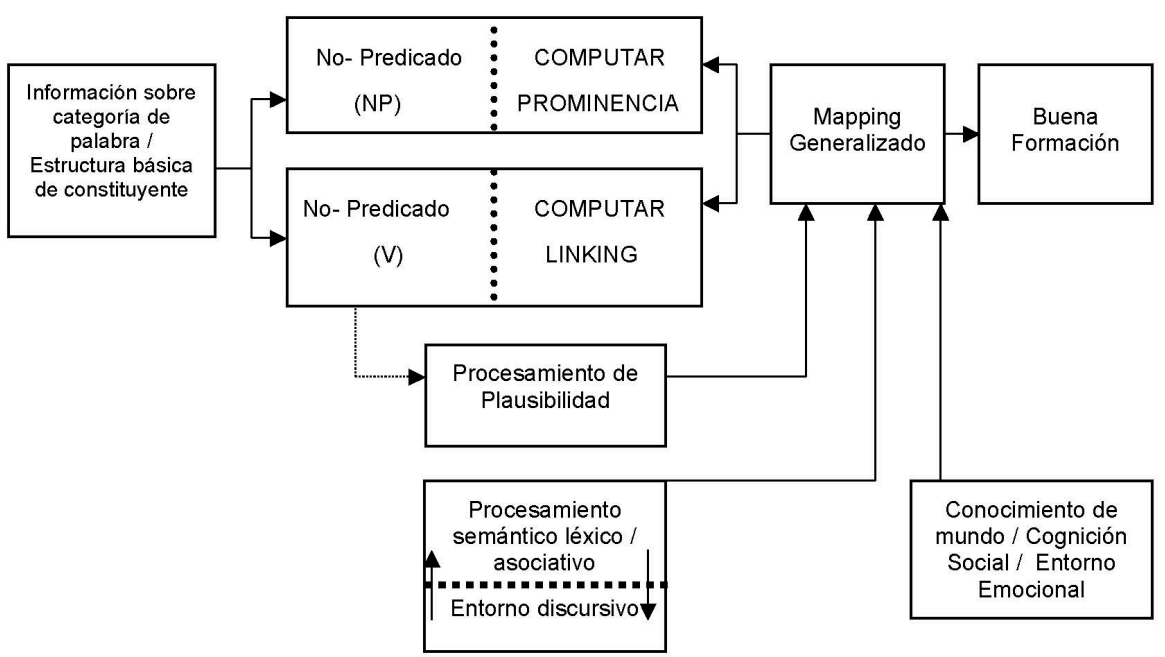

Figura 1: La arquitectura de la última versión del Extended Argument Dependency Model (eADM; adaptado de Bornkessel-SchlesewskyySchlesewsky 2008).

La computación del estatus de prominencia de un argumento se basa en distintos rasgos lingüísticos, cuya informatividad depende de la lengua bajo consideración. Uno de los rasgos con una fuerte influencia a nivel interlingüístico es la animacidad. Estudios previos sobre el alemán han demostrado que la animacidad de un argumento interactúa con la marca morfológica de caso (Frisch y Schlesewsky 2001, Roehm et al. 2004). La presentación de preguntas con un argumento inicial animado con marca de caso acusativa y la presentación de un segundo argumento inanimado engendra una negatividad centro-parietal (N400) ${ }^{1}$ en la posición de la segunda frase nominal (FN) a comparación de una FN animada en la misma posición. Según Bornkessel y Schlesewsky (2006), este efecto es el resultado de un incremento en el costo de procesamiento asociado con tener que asignar el rol
1. Los principales componentes electrofisiológicos relacionados con la aparición de un estímulo lingüístico que tienen lugar en los estudios bajo el paradigma de potenciales evocados por eventos (o ERPs por sus siglas en ingles) son el ELAN, el N400 y el P6oo. El componente ELAN Ileva su nombre de acuerdo con las siglas en ingles referentes a Early Left Anterior Negativity que señalan la temporalidad (temprana, con un pico cerca de los 150 milisegundos), la localización en la corteza cerebral (izquierda anterior) y la forma de onda (negativa) en comparación con un estímulo de referencia. Este componente tiene lugar ante la aparición de una palabra que no está permitida por la sintaxis de la lengua. Por ejemplo, ante la aparición de la preposición "de" en "El gato salió por de la ventana". Generalmente va acompañado del componente P6oo (Friederici 2002).Como su nombre lo indica, el $\mathrm{N}_{4} \mathrm{Oo}$ tiene un pico cerca de los 400 milisegundos luego de la aparición del estímulo y es negativo en relación con la onda eléctrica generada por el estímulo que se considera como referencia. Suele tener una distribución centro-parietal en la corteza cerebral y tiene lugar ante la aparición de incongruencias semánticas del tipo "El cocinero batió la mezcla con una escopeta" frente al sustantivo "espátula" (Kutas y Hillyard1983), y también frente a la aparición de un estímulo que es semánticamente posible pero inesperado: "El cocinero batió la mezcla con una amiga" frente a "batidora" o "cuchara" que serían más esperados. Se lo interpreta como un componente relacionado con el costo de integración de la información semántica entrante. Por ultimo, el P6oo es un componente tardío, cuyo pico ronda los 600 milisegundos. Tiene una distribución amplia a lo largo de toda la corteza cerebral, y la forma de onda es positiva con respecto a la onda generada por un estímulo de referencia. Aparece ante incongruencias relacionadas con el análisis sintáctico de una oración, como se $\mathrm{da}$, por ejemplo, en las oraciones de vía muerta del tipo "The horse past the fence fell", en las que el procesador de lenguaje elige un análisis sintáctico posible y luego (ante la aparición de fell) se da cuenta de que la estructura elegida es incorrecta. También tiene lugar ante incongruencias generadas por ambigüedad de caso, o de concordancia, entre otras. Se lo interpreta como la marca electrofisiológica relacionada con el costo de integración y de reparación sintáctica, aunque también se han encontrado casos en los que aparece ante incongruencias semántica (Kim y Osterhout 2005). 
de 'Actor' a un argumento inanimado. De manera más precisa, siguiendo el algoritmo de enlace propuesto por RRG, cuando a una frase con marca de caso acusativo se le asigna el rol de 'Undergoer', la aplicación de las jerarquías de prominencia dan lugar a la predicción de la aparición de un 'Actor'. Cuando aparece la segunda FN inanimada, no se completa la correlación, y la asignación de un estatus más prominente a este argumento resulta más costoso, tal como es reflejado por el N400. Efectos similares han sido encontrados en inglés (Weckerly y Kutas 1999) debido a la interacción entre animacidad y posición del argumento, y en italiano (Dröge, Maffongelli y Bornkessel-Schlesewsky 2014) debido a la modulación de orden oracional y tipo de verbo. Por otro lado, en chino, una lengua que carece de marca de caso, la animacidad interactúa con el número de argumentos disponibles previos a la aparición del verbo. Los datos muestran que la interacción entre animacidad e interpretación del argumento (como sujeto o como objeto oracional) evoca una negatividad anterior cuando el verbo está precedido por dos argumentos (Wang et al. 2012), mientras que no evoca ningún efecto cuando el verbo está precedido por un solo argumento ambiguo (Wang et al. 2009). Es decir que, según muestra la evidencia que apoya al modelo de Bornkessel y Schlesewsky, una falla en la computación de la prominencia puede generar efectos similares aunque las estructuras a comparar en distintas lenguas sean diferentes, y efectos diferentes aunque las estructuras a comparar sean similares. La conclusión es que el procesamiento es altamente sensible a las características formales de cada lengua.

\subsubsection{Procesamiento de verbos}

Cuando el procesador encuentra e identifica un verbo (fase 1), en la primera parte de la fase 2 se extraen su estructura lógica, voz y rasgos de concordancia. En la fase $2 b$, se integra el verbo con toda la información previa disponible correspondiente a los argumentos de la oración. En consecuencia, se establecen las relaciones de concordancia (en la etapa establecer concordancia) y el enlace de los argumentos (i.e., la interpretación de los argumentos a través de la asociación de cada argumento previo con una posición en la estructura lógica del verbo). El paso establecer concordancia es probablemente el proceso más examinado en términos de evidencia neurofisiológica a nivel interlingüístico (cf., por ejemplo, de Vincenzi, Job, Di Matteo, Angrilli, Penolazzi y Ciccarelli 2003, en italiano; Hagoort y Brown 2000, en holandés; Osterhout y Mobley 1995, en inglés; y Roehm, Bornkessel, Haider y Schlesewsky 2005, en alemán). La evidencia muestra que en lenguas como el italiano o el inglés, si el sistema de procesamiento encuentra un verbo que no concuerda con los rasgos de concordancia del argumento precedente, se genera una negatividad anterior izquierda (LAN, por sus siglas en ingles correspondientes a Left Anterior Negativity). Estudios similares en alemán han mostrado que si se manipula la ambigüedad de caso del argumento precedente, no se genera un efecto de tipo LAN, sino que se evidencian efectos del tipo P600 (beim Graben et al. 2000).

Además de activar y computar los rasgos de concordancia del verbo, en esta segunda fase se computa el tipo de enlace (linking) que se establece entre la jerarquía de prominencia y la estructura lógica del verbo. Para la realización de este paso, el procesador toma la información sobre Roles Semánticos Generalizados (RGs: 'Actor' o 'Undergoer'), concordancia de los argumentos previos ([+agrt] o [-agrt]) y la voz del evento (voz activa o 
pasiva). Si la asignación de RGs no fue realizada antes de que se procese el verbo (debido a la ambigüedad de caso de los argumentos, por ejemplo), esta se realiza en el paso computar linking. La evidencia en alemán muestra que un desajuste entre el tipo de enlace predicho en el paso computar prominencia y el tipo de enlace requerido por la estructura lógica del verbo se traduce en una positividad parietal temprana, o P345 (Bornkessel et al. 2002a, 2003, en alemán; Lamers 2001, en holandés); o en una positividad tardía del tipo P600 (Dröge et al. 2014, en italiano).

\subsubsection{Fase 3}

A continuación del procesamiento de elementos del centro realizado en la fase 2, en la fase 3 se realiza el mapeo generalizado de todos los tipos de información disponibles, para realizar una evaluación de buena formación y para la reparación en caso de haber algún conflicto en el procesamiento.

\subsubsection{Mapeo generalizado}

El paso de mapeo generalizado de la fase 3 integra todas las fuentes de información lingüística disponibles, tanto internas al centro (core) de la cláusula como externas. En particular, el eADM asume que factores como la prosodia, la plausibilidad, el conocimiento de mundo, la frecuencia de ocurrencia y la actualización semántica no modulan el procesamiento de las relaciones del centro (core) en la fase 2. La influencia de estos factores observada en experimentos comportamentales (Kempen y Harbusch 2005; McRae, Ferretti y Liane Amyote 1997) es el resultado de problemas en el mapeo generalizado de la fase 3 , en la que se integran las informaciones de salida del procesamiento de estructuras centrales y no centrales provenientes de la fase 2. El modelo predice que la manipulación de estos tipos de información conduce a una modulación del componente positivo tardío (del tipo P600: Kaan, Harris, Gibson y Holcomb 2000; Friederici, Steinhauer, Mecklinger y Meyer 1998).

\subsubsection{Buena formación / Reparación}

Una vez realizado el paso de mapeo generalizado, el sistema de procesamiento evalúa la buena formación del input, y repara el procesamiento en caso de ser factible. El modelo asume que puede establecerse una distinción entre los componentes del tipo P600 correspondientes al reanálisis de funciones gramaticales (como ocurriría, por ejemplo, en caso de que haya un error en concordancia en la fase $2 \mathrm{~b}$ ) y las positividades tardías observadas en estructuras mal formadas. Los autores señalan que estas últimas constituyen un reflejo de la evaluación de buena formación / pasos de reparación en la fase 3 (De Vincenzi et al. 2003; Hagoort, Brown y Groothusen 1993; Neville et al. 1991; Osterhout y Holcomb 1992, 1993). También aclaran que si bien es necesario un estudio más sistemático sobre este tipo de disociación para poder establecer esta separación teórica, existe evidencia neuroanatómica que apoya la distinción entre el procesamiento de oraciones que involucran una violación sintáctica y oraciones que involucran una estructura oracional no preferida que también genera positividades tardías (Friederici 2004). Los datos muestran además que los efectos positivos deberían ser interpretados como el reflejo de procesos más 
generales relacionados con la evaluación de buena formación, en vez de reflejar solamente un incremento en el esfuerzo generado por el procesamiento sintáctico. Por ejemplo, Gunter, Stowe y Mulder (1997, experimento 2) observaron una modulación de una positividad tardía como respuesta a una manipulación semántica. Además, Steinhauer, Mecklinger, Friederici y Meyer (1997) observaron que las positividades tardías pueden ser moduladas por manipulaciones internas al experimento relacionadas con la probabilidad de ocurrencia de las estructuras con una aceptabilidad degradada (cf. también Hahne 1997). Roehm (2004) reporta un componente bifásico del tipo N400-positividad tardía al procesar oraciones como The opposite of black is nice ('Lo opuesto de negro es lindo'). Aquí tampoco hay problemas en el procesamiento sintáctico, sino que los efectos positivos aparecen como el resultado de la evaluación global de la buena formación de la oración.

\subsection{Presupuestos generales del Extended Argument Dependency Model}

Además de proponer la existencia de distintas fases de procesamiento que se corresponden con correlatos neurofisiológicos y neuroanatómicos diferenciados el eADM postula lo siguiente:

(i) La comprensión es alcanzada luego del completamiento de diferentes etapas. Es serial.

(ii) El procesador no espera hasta el final de la oración para comenzar con la interpretación, sino que integra cada elemento tan pronto como está disponible a la vez que genera predicciones sobre los elementos que vendrán. Es incremental (Kamide 2008; Kutas, DeLong y Smith 2011; Van Berkum et al. 2005; Wicha, Moreno y Kutas 2004).

Una de las principales motivaciones para postular este modelo es explicar cuáles son los aspectos lingüísticos relevantes a la hora de predecir - sobre todo en lenguas de verbo final-cuál será el tipo de verbo, y en consecuencia de linking entre sintaxis y semántica, que tendrá lugar en la oración. A diferencia de otros modelos seriales (Frazier y Fodor 1978, Friederici 1998, Friederici 2002), la noción de predictibilidad para el eADM no sólo implica la generación de una expectativa con respecto a la estructura sintáctica de los elementos que vendrán, sino también a su interpretación semántica. Esto quiere decir que, incluso ante la ausencia de información verbal, a los argumentos se les asigna un rol semántico generalizado (i.e., 'Actor' o 'Undergoer') de acuerdo con su prominencia relativa (fase 2b: computar prominencia). En cuanto a los verbos, su procesamiento implica que la representación léxica de los argumentos verbales (su estructura lógica, [el] en la figura 1) sea asociada a los argumentos que ya han sido procesados mediante la computación de las relaciones de prominencia, concordancia y voz (activa, pasiva; paso computar linking; Bornkessel y Schlesewsky 2006). Otra de las características del eADM es que asume que el procesador sigue un principio de 'menor esfuerzo posible', también denominado 'Minimalidad', que se basa en la idea de que se asume la estructura sintáctica y la interpretación más simple compatible con los elementos que ya han aparecido. De esta manera, el modelo provee predicciones para el procesamiento de oraciones en alemán con argumentos con marca de caso ambigua. Con respecto a la asignación de roles semánticos, el modelo postula una ventaja para la interpretación del argumento nominativo con el rol más alto en la jerarquía temática (i.e., 'Actor'), tal como lo postula RRG (Bornkessel-Schlesewsky y Schlesewsky 2009a).

Por último, es necesario tener en cuenta que este modelo entiende el procesamiento de lenguaje como un proceso interactivo, en el sentido de que 
en primer lugar es guiado por las limitaciones sintácticas (i.e., cada palabra de una oración desencadena la activación de una serie de esquemas sintácticos), pero que rápidamente se vale de la información sobre jerarquización de los argumentos provista por características morfosintácticas y rasgos específicos del lenguaje sobre animacidad y definitud para computar la prominencia de los argumentos y generar expectativas sobre las palabras que podrían aparecer.

\section{Linking and Checking Model (LCM)}

El Linking and Checking Model (LCM) es un modelo de procesamiento que basa sus supuestos en el modelo minimalista del generativismo (Chomsky 1993, 1995, 2000). El modelo apela a la extensa cantidad de evidencia obtenida en alemán para dar cuenta de algunos fenómenos cognitivos que se dan durante el procesamiento de oraciones en esta lengua con ambigüedad de caso. Al igual que el eADM, el LCM es un modelo serial e incremental. Sin embargo, las predicciones sobre el tipo de información que aparecerá dada una cadena de palabras determinada provienen de manera exclusiva de la información sintáctica de los elementos que aparecieron con anterioridad. Es decir que el modelo no tiene en cuenta la interacción de factores provenientes de la semántica para la interpretación de significado.

\subsection{Estructura del modelo}

Como lo muestra la figura 2, el modelo de Bader y Bayer también se basa en una serie de etapas después de las cuales la oración puede ser comprendida. El LCM postula que el Mecanismo de Procesamiento de Oraciones Humano (HSPM, por sus siglas en inglés) está fraccionado de la siguiente manera: en primer lugar, se llevan a cabo procesos de ensamblaje de estructuras, que computan la estructura sintáctica de las estructuras de frase. Luego, se dan los procesos de enlace, que asocian las frases dentro de la estructura de frase con las posiciones de una estructura argumental posible. Por último, se da el proceso chequeo, en el que se coteja la distribución correcta de los rasgos de caso y la concordancia entre verbo y sujeto oracional. A continuación se exponen las características de cada uno de estos procesos.

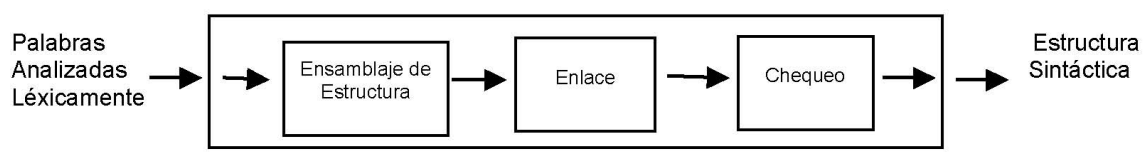

Figura 2: La arquitectura del Linking and Checking Model (LCM; adaptado de Bader y Bayer 2006).

\subsubsection{Ensamblaje de estructuras}

El ensamblaje de estructuras sintácticas consiste en la construcción de estructuras de frase a partir de la información sintáctica provista por el Marcador de Frase Parcial Actual (CPPM, por sus siglas en inglés), y se realiza en concordancia con algunos preceptos de la teoría generativista. Básicamente, las operaciones que tienen lugar en esta primera etapa son la operación de 'Ensamble' (Merge, en inglés) y la operación 'Muévase $\alpha$ ' (Move $\alpha$ ). La primera constituye la operación sintáctica mínima mediante la cual se construyen las relaciones jerárquicas que subyacen a la estructura 
de una oración. La segunda es la regla que subsume a todas las transformaciones de movimiento, y que implica que cualquier constituyente puede ser trasladado o movido de la posición que ocupa en la estructura sintáctica. De manera análoga a estas dos operaciones sintácticas, los principios de procesamiento de estructura de frase pueden ser clasificados o bien como principios de adjunción de estructuras, o bien como principios que permiten procesar estructuras de dependencia (filler-gap dependencies), donde hay un antecedente y una huella sintáctica.

La construcción de estructuras de frase se realiza, además, de acuerdo con los siguientes principios:

(i) Simplicidad. El procesador no construirá estructuras vacuas (Gorrell 1995).

(ii) Adjunción Mínima. El procesador evitará postular estructuras de frase innecesarias para la estructura superficial, pero no demorará el procesamiento de las estructuras de frase requeridas (De Vincenzi 1991).

(iii) Preferencia de Caso. Si es posible, preferir caso nominativo al caso acusativo. (El dativo es analizado como una frase de caso y es, por lo tanto, diferente a los otros casos y menos preferido debido al principio de simplicidad.)

\subsubsection{Enlace}

El LCM se centra en las segundas dos etapas del procesamiento. Como se describió anteriormente, en el proceso de enlace, se asocian las frases del Marcador de Frase Parcial Actual con las posiciones de una estructura argumental posible. Basándose en evidencia obtenida a través de distintos experimentos en alemán, los autores señalan que el proceso de enlace se realiza de acuerdo con el Principio de Enlace de Argumento que consta de dos pasos:

(i) Enlazar la Frase Determinante (FD) nominativa del Marcador de Frase Parcial Actual con el argumento estructuralmente más alto, y la FD de objeto al argumento restante.

(ii) Si el paso anterior conduce a un conflicto respecto de la animacidad y si invertir el enlace elimina el conflicto, revertir el enlace.

Por último, cuando el verbo tiene tres argumentos, como ocurre en los verbos ditransitivos, el enlace se realiza de la misma manera, y se asigna, jerárquicamente, el objeto con caso dativo al último argumento de la oración.

\subsubsection{Chequeo}

De acuerdo al Algoritmo de Chequeo Basado en el Enlace (Linking-Based Checking Algorithm, en inglés), la primera tarea del HSPM una vez que enlaza una FD a un argumento consiste en chequear si la FD tiene el tipo de rasgos requeridos por su posición argumental (de caso y de número) o no. Si el chequeo de rasgos tiene como resultado una incongruencia con lo requerido por la estructura argumental, la siguiente tarea del HSPM será determinar si el Marcador de Frase Parcial Actual puede ser reparado localmente a través del reemplazo de los rasgos incorrectos por los correctos o no. Esta reparación se realizaría posiblemente con un reajuste automático del Marcador de Frase Parcial Actual. Estas dos tareas cercanamente relacionadas - chequeo y reparación de rasgos-constituyen el segundo paso del Algoritmo de Chequeo. La figura 3 muestra los pasos más relevantes del Algoritmo de Chequeo Basado en el Enlace. 
1. Enlace de Argumento

Enlazar cada FD dentro del Marcador de Frase Parcial Actual a una posición dentro de la Estructura argumental del verbo.

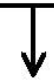

\section{Manipulación de Rasgos}

\section{A. Chequeo de Rasgos}

Chequear los rasgos relevantes (caso para los objetos y número y persona para el sujeto).

\section{B. Reparación de rasgos}

Para cada incongruencia resultante, donde la incongruencia tiene la forma "valor de rasgo a asignado a una $\mathrm{FX}$ en lugar de un valor de rasgo $\beta$ ", determinar si el material léxico de la FX sería compatible con la asignación de $\beta$.

Si es así, reemplazar $\alpha$ por $\beta$ y - si es necesarioajustar el marcador de frase de manera acorde.

Figura 3: El Algoritmo de Chequeo Basado en el Enlace (adaptado de Bader y Bayer 2006).

\subsection{Presupuestos generales del Linking and Checking Model}

Al igual que el modelo eADM, el LCM asume que el Mecanismo de Procesamiento de Oraciones Humano es de tipo serial, es decir que se completa tras la sucesión de distintas etapas. Los autores del modelo asumen que el mecanismo de procesamiento puede ser descompuesto en distintos componentes, y que uno de ellos (en el que centran sus aportes) está restringido a la tarea de realizar el enlace y el chequeo de rasgos sintácticos. Además, estas dos tareas están ordenadas de manera estricta. Primero se enlazan los argumentos, y luego se aplican operaciones de chequeo al resultado de las operaciones de enlace. Esta es una diferencia fundamental entre los dos modelos expuestos. En el eADM, el enlace se realiza después de la computación de los rasgos sintáctico-semánticos, en la fase de mapeo generalizado.

Otra diferencia crucial entre ambos modelos es el rol que tienen los rasgos semánticos durante la realización del enlace entre sintaxis y semántica. Mientras que el eADM postula la interacción de distintos factores (tanto sintácticos como semánticos) que permiten predecir la aparición de un input determinado que se va actualizando a medida que aparecen otros tipos de información, el LCM propone que el procesador no utiliza ningún tipo de información semántica para realizar el enlace inicial, y que sólo se recurre a los rasgos de animacidad en caso de que se dificulte el procesamiento. Por ejemplo, considérese el ejemplo (2): 
(2) Keiner wußte, daß Britta das Buch gefiel

Nadie saber ${ }_{\text {[PAS-rra-SG] }}$ que Britta $_{\text {[DAT] }} e_{\text {[NOM] }}$ libro $_{\text {gustar }}{ }_{\text {[PAS-3ra-SG] }}$

'Nadie sabía que a Britta le gustaba el libro'.

El Marcador de Frase Parcial Actual contiene dos FDs. Debido al Principio de Preferencia de Caso, a la primera FD se le asigna caso nominativo, y a la segunda FD, caso acusativo. De acuerdo con el Principio de Enlace Argumental, el procesador en primer lugar tratará de enlazar el argumento referido al estímulo que genera la emoción a la primera FD marcada con caso nominativo, Britta, y al experimentante con el segundo argumento con marca acusativa, das Buch ('el libro'). Como este patrón particular de enlace conduce a una violación causada por la animacidad del argumento — das Buch es inanimado y por lo tanto no puede ser el experimentante del evento-, la segunda cláusula del Principio de Enlace Argumental requiere al procesador que invierta el enlace inicial. El resultado puede verse en la figura 4, adaptada de Bader y Bayer (2006).

\section{a. Estructura que resulta luego del Paso 1 y Paso $2 \mathrm{~A}$}

b. Paso 2B
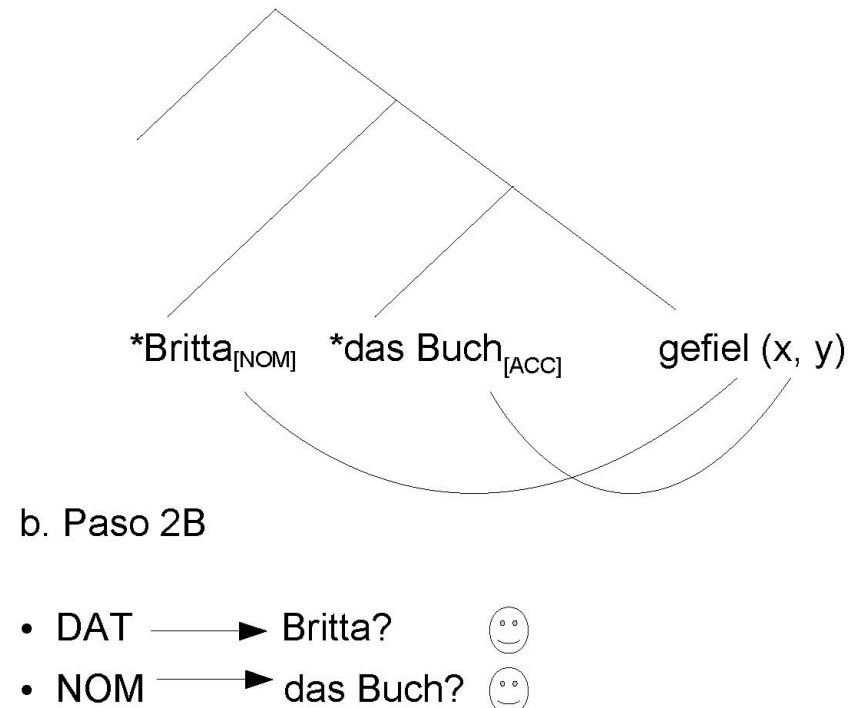

Figura 4: Ejemplo de procesamiento de oración con argumento nominativo inanimado (adaptado de Bader y Bayer 2006).

Una inspección de (4a) muestra que el enlace entre el Marcador de Frase Parcial Actual y la estructura argumental ahora está bien formado en términos semánticos pero no en términos sintácticos. Cuando se comparan los rasgos de Caso requeridos por la estructura argumental con la marca de caso otorgada a las FDs, aparecen dos violaciones de caso: la primera FD tiene caso nominativo en vez de dativo - tal como lo requiere el verbo-, y la segunda FD tiene caso acusativo en lugar de caso nominativo. Aquí se aplica el segundo punto del Principio de Enlace Argumental. Para las dos FDs que incurren en la violación de caso, el procesador tiene que determinar si estas FDs también serían compatibles con los rasgos de caso requeridos por la estructura argumental del verbo. Es decir, tiene que chequear si la FD Britta es morfológicamente compatible con el caso dativo, y si la FD das Buch es morfológicamente compatible con caso nominativo. La respuesta 
es afirmativa en ambos casos, y el procesador sólo tiene que reemplazar los rasgos de caso asignados en primera pasada por los rasgos de caso prescriptos por la estructura argumental del verbo.

Otra de las características que comparten el LCM y el eADM es que ambos modelos se postulan como incrementales, es decir que el procesador actualiza sus hipótesis acerca de lo que está procesando -y sobre la información que aparecerá a continuación- de acuerdo con información lingüística previa. La diferencia entre ambos modelos es, nuevamente, que el LCM postula que en una primera instancia - en los procesos correspondientes al procesamiento de primera pasada (first-pass parsing), la información que tiene más relevancia para el procesamiento es la información sintáctica (marca de caso en los objetos; número y persona en el caso del sujeto). En cambio, el eADM incluye los rasgos de 'animacidad', y 'definitud' como rasgos fundamentales para establecer el enlace desde un comienzo.

Por último, ambos modelos postulan la existencia de un mecanismo de Simplicidad/Minimalidad que, ante la posibilidad de procesar una estructura de varias formas posibles, opta por la estructura más sencilla. Cabe aclarar que, si bien ambos modelos entienden este mecanismo de mínimo esfuerzo tal como lo postularon Fodor (1998) e Inoue y Fodor (1995), en el caso del LCM este mecanismo tiene lugar en la primera etapa de procesamiento (ensamblaje de estructuras), mientras que en eADM este principio puede ser aplicado en etapas posteriores, como computar prominencia (fase $2 \mathrm{~b}$ ), y la computación de la estructura más simple posible puede depender, en consecuencia, de la información tanto sintáctica como semántica de los argumentos y del verbo.

\section{Explicaciones del eADM y el LCM ante el mismo fenómeno}

Tanto eADM como LCM tienen supuestos en común, como el principio de Minimalidad/Simplicidad, el hecho de que los modelos inicien el procesamiento con la interpretación de la información sintáctica, y el rol que le dan a la marca de caso para el enlace entre sintaxis y semántica. Sin embargo, sostienen diferencias significativas, en relación al tipo de información que interviene, durante el mapeo de la información sintáctica y semántica, en la comprensión en tiempo real. En consecuencia, las predicciones que se desprenden de uno y otro modelo varían de manera acorde. Por ejemplo, Bornkessel et al. (2003) analizan el procesamiento de oraciones con verbo final que requieren una inversión del enlace entre sintaxis y semántica cuando los participantes leen el verbo similares a las que aparecen en (1). Los autores manipularon el orden oracional (SOV y OSV) y el tipo de verbo de la cláusula subordinada (Verbo de Actividad vs. Verbo Psicológico con Objeto Experimentante), y encontraron que durante la lectura de la primera región (primer argumento) no había diferencias en la modulación de los potenciales entre las oraciones con sujeto y con objeto iniciales. En la segunda región, los autores encontraron la aparición de una positividad temprana en las condiciones que comenzaban con Objeto. Por último, independientemente del orden, la aparición de un verbo con Objeto Experimentante produjo una positividad entre los 300 y los 600 ms. luego de la aparición del verbo.

Recordemos que el eADM asume que el procesador computa la relación jerárquica entre argumentos apostando a una relación que comienza con un argumento más prominente y termina con uno menos prominente. Según 
este modelo, la falta de diferencia en el primer argumento se debe a que el alemán permite estructuras pasivas con objeto con marca de caso dativo inicial, tal como se ejemplifica en (3):

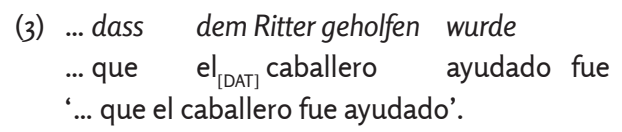

Como se describió en la sección 3.1, tras la activación de la información de clase de palabra (fase 1) el sistema tiene en cuenta una serie de plantillas sintácticas legales en la lengua, y elige la más sencilla mediante el principio de minimalidad en base a la interacción de esta información y la información computada en la segunda fase (computar prominencia). Como la información morfológica y de prominencia no ofrecen ningún problema, el objeto es enlazado con un posible único argumento (no macrorrol) en una potencial estructura lógica de un verbo en voz pasiva, y el mapeo generalizado se realiza sin interrupción alguna.

Con la aparición del segundo argumento con caso nominativo, la predicción sobre el tipo de enlace y la jerarquía de prominencia del primer argumento debe ser corregida, proceso que da lugar a la positividad temprana observada. El reanálisis de la escala de prominencia deriva en un argumento dativo (no macrorrol) y un argumento nominativo, enlazado con el 'Actor' del evento. La aparición de un verbo con Objeto Experimentante da lugar a un nuevo reanálisis y una nueva positividad, esta vez, debido a una reasignación de roles temáticos para el argumento nominativo (de 'Actor' a 'Undergoer').

Por su parte, el LCM sostiene que hay una diferencia primordial entre las oraciones con verbos de actividad y verbos psicológicos con Objeto Experimentante, que radica en la naturaleza de la alternancia en el orden oracional de las oraciones con verbos de actividad y con verbos psicológicos con Objeto Experimentante. Basándose en los preceptos generativistas sobre movimiento sintáctico, el orden oracional en una oración como (4), esto es OSV, está motivado a través de la noción de scrambling, es decir, el resultado de un movimiento-A' a través de la adjunción de una XP a una IP o VP (Kosta 2006).

(4) Maria glaubt...

María creer[3ra-SG]...

'María cree...'

$\begin{array}{llll}\text {... dass } & \text { dem Priester } & \text { der Gärtner } & \text { folgt. } \\ \ldots \text { que } & \mathrm{el}_{[\mathrm{DAT}]} \text { cura } & \mathrm{el}_{[\mathrm{NOM}]} \text { jardinero } & \text { seguir }_{[3 \mathrm{ra}-\mathrm{SG}]^{\circ}}\end{array}$

'... que al cura sigue el jardinero'.

Por otro lado, el LCM asume que el orden de palabras en el grupo de verbos psicológicos con Objeto Experimentante está generado en la base, es decir que el orden oracional está motivado léxicamente, y no por un movimiento hacia la izquierda dentro de la proyección (extendida) del verbo (Bader y Bayer 2006, 52). Como consecuencia, en la posición de V' no se deja una huella, no se establece una dependencia de larga distancia y el procesamiento de oraciones con objeto inicial y verbos psicológicos con Objeto Experimentante debería ser mucho más fácil que el procesamiento de las oraciones con orden OSV y verbos de actividad. El modelo predice, además, que no debería haber diferencias entre el procesamiento de oraciones con sujeto y con objeto inicial y verbos con Objeto Experimentante. 
De esta diferencia en el tipo de explicaciones dadas por ambos modelos, se pueden analizar varias cuestiones: primero, los resultados de Bornkessel et al. (2003) muestran que la primera predicción de LCM no se cumple. Durante el procesamiento incremental de las oraciones con orden OSV no se ve una diferencia entre las oraciones con verbos de actividad y con Objeto Experimentante que dependa exclusivamente del tipo de verbo, sino que las diferencias producidas por el tipo de verbo se dan tanto para las oraciones con orden SOV como para las que siguen el orden OSV. Segundo, en cuanto al poder explicativo de ambos modelos, LCM falla al explicar cuál es el mecanismo que permite estipular qué clase de verbos se comportan como los verbos de Actividad y cuáles se comportan como los verbos con Objeto Experimentante y por qué se daría esta división en la clase de verbos que toman tal o cual mecanismo para determinar cuál es su orden de base. Para un modelo sobre procesamiento de lenguaje, esto implica que el sistema debe basar las predicciones sobre el tipo de enlace que tendrá lugar durante la comprensión de un evento tanto en reglas que responden a principios universales del procesamiento de lenguaje como a excepciones. A través del paso de computar prominencia y de la inclusión de rasgos semánticos como piezas de información fundamental en este paso, eADM logra sortear esta falla y explicar mayor cantidad de evidencia psicolingüística. En este modelo, la prominencia da cuenta de la relación jerárquica entre dos o más argumentos en una cláusula. La información utilizada para establecer el grado de prominencia de un constituyente en relación a otro constituyente comprende el rol temático, la marca morfológica de caso, la posición, la animacidad, la persona y la definitud del argumento entre los rasgos más salientes. Este constructo le permite al eADM dar cuenta de la variabilidad entre lenguas, en tanto que cada una da mayor peso a una u otra jerarquía para establecer el grado de prominencia de los argumentos. Además, le permite establecer un principio común a la interpretación argumental en tiempo real que se deriva de toda la evidencia psicolingüística hallada hasta el momento en lenguas como el alemán (Bornkessel, Schlesewsky y Friederici 2002b, 2003; Bornkessel et al. 2005; Bornkessel-Schlesewsky y Schlesewsky 2009a), el chino (Wang et al. 2009, Wang et al. 2012), el holandés (Lamers 2001), el inglés (Weckerly y Kutas 1999), el finés, el ruso (Frisch, Schlesewsky y Wegner 2005), el italiano (Dröge, Maffongelli y Bornkessel-Schlesewsky 2014) y el español (Gattei, Dickey et al. 2015; Gattei et al. 2017; Gattei, Tabullo et al. 2015): el procesador tiende a asignar el rol temático con mayor grado de actividad (el 'Actor') lo más rápido posible, y predice el tipo de verbo (y por lo tanto el tipo de enlace entre sintaxis y semántica que tendrá lugar en consecuencia.

\section{Conclusión}

En este trabajo rescatamos un tipo de fenómeno que pone de relevancia la interacción entre el enlace y el orden de palabras para contrastar las predicciones de dos modelos psicolingüísticos alternativos sobre datos experimentales conocidos. El fenómeno involucra un tipo de enlace no canónico en alemán pero típico en los verbos psicológicos tal que un Experimentante se realiza en una frase nominal marcada con caso dativo mientras que el Estímulo se realiza mediante una frase nominal nominativa. A su vez, este enlace tiene lugar en una estructura con orden OSV.

El procesamiento de este tipo de oraciones genera distintos potenciales que las teorías deberían predecir. Se concluye que el modelo eADM fuertemente 
basado en la teoría gramatical del Rol y la Referencia predice correctamente tales potenciales mientras que el modelo LCM basado en la teoría Minimalista de tradición Generativista no hace todas las predicciones de manera satisfactoria. Identificamos, además, que el factor diferenciador de las dos teorías con mayor incidencia en motivar las diferentes predicciones es que LCM retarda el uso de información no sintáctica hasta tener un esquema sintáctico completo. Por el contrario, eADM permite al procesador recurrir a información semántica incluso sobre la base de una descripción sintáctica muy parcial y escueta. 


\section{Q Bibliografía}

" Altmann, Gerry T. M. y Yuki Kamide. 1999. "Incremental interpretation at verbs: Restricting the domain of subsequent reference". Cognition 73.3: 247-264.

" Arai, Manabu y Frank Keller. 2013. “The use of verb-specific information for prediction in sentence processing”. Language and Cognitive Processes 28.4: 525-560.

"Bader, Markus y Josef Bayer. 2006. Case and linking in language comprehension: Evidence from German. Studies in Theoretical Psycholinguistics 34. Dordrecht: Springer.

" beim Graben, Peter, J. Douglas Saddy, Matthias Schlesewsky y Jürgen Kurths. 2000. "Symbolic dynamics of event-related brain potentials". Physical Review E 62.4: 5518.

" Bornkessel, Ina y Matthias Schlesewsky. 2006. "The extended argument dependency model: A neurocognitive approach to sentence comprehension across languages". Psychological review 113.4: 787.

» Bornkessel, Ina, Matthias Schlesewsky y Angela D. Friederici. 200za. “Beyond syntax: Language-related positivities reflect the revision of hierarchies". NeuroReport 13.3: 361-364.

" Bornkessel, Ina, Matthias Schlesewsky y Angela D. Friederici. 2002b. “Grammar overrides frequency: Evidence from the online processing of flexible word order". Cognition 85.2: B21-B30.

» Bornkessel, Ina, Matthias Schlesewsky y Angela D. Friederici. 2003. "Eliciting thematic reanalysis effects: The role of syntax-independent information during parsing”. Language and Cognitive Processes 18.3: 269-298.

» Bornkessel, Ina, Stefan Zysset, Angela D. Friederici, D. Yves von Cramon y Matthias Schlesewsky. 2005. "Who did what to whom? The neural basis of argument hierarchies during language comprehension". Neuroimage 26.1: 221-233.

" Bornkessel-Schlesewsky, Ina y Matthias Schlesewsky. 2008. "An alternative perspective on 'semantic P600' effects in language comprehension". Brain Research Reviews 59.1: $55-73$.

"Bornkessel-Schlesewsky, Ina y Matthias Schlesewsky. 2009a. "Minimality as vacuous distinctness: Evidence from cross-linguistic sentence comprehension”. Lingua 119.10: 1541-1559.

»Bornkessel-Schlesewsky, Ina y Matthias Schlesewsky. 2009b. Processing syntax and morphology: A neurocognitive perspective. Oxford: Oxford University Press.

"Brown, Colin y Peter Hagoort. 1993. "The processing nature of the N400: Evidence from masked priming". Journal of Cognitive Neuroscience 5.1: 34-44.

"Chomsky, Noam. 1993. Lectures on government and binding: The Pisa lectures. Berlin, New York: Walter de Gruyter.

»Chomsky, Noam. 1995. The minimalist program. Cambridge, MA: MIT Press.

"Chomsky, Noam. 2000. New horizons in the study of language and mind. Cambridge: Cambridge University Press.

"Chwilla, Dorothee J. y Herman H. J. Kolk. 2005. “Accessing world knowledge: evidence from N400 and reaction time priming". Cognitive Brain Research 25.3: 589-606.

»De Vincenzi, Marica. 1991. Syntactic parsing strategies in Italian: The minimal chain principle. Studies in Theoretical Psycholinguistics 12. Dordrecht: Springer. 
"De Vincenzi, Marica, Remo Job, Rosalia Di Matteo, Alessandro Angrilli, Barbara Penolazzi, Laura Ciccarelli y Francesco Vespignani. 2003. "Differences in the perception and time course of syntactic and semantic violations". Brain and Language 85.2: 280-296.

"DeLong, Katherine A., Laura Quante y Marta Kutas. 2014. "Predictability, plausibility, and two late ERP positivities during written sentence comprehension". Neuropsychologia 61: 150-162.

》Dröge, Alexander, Laura Maffongelli e Ina Bornkessel-Schlesewsky. 2014. “Luigi piace a Laura? Electrophysiological evidence for thematic reanalysis with Italian dative object experiencer verbs". En Structuring the Argument: Multidisciplinary research on verb argument structure, editado por Asaf Bachrach, Isabelle Roy y Linnaea Stockall, 83-118. Amsterdam: John Benjamins.

" Ferreira, Fernanda y John M. Henderson. 1991. "Recovery from misanalyses of gardenpath sentences". Journal of Memory and Language 30.6: 725-745.

" Fodor, Janet Dean. 1998. “Learning to parse?”. Journal of Psycholinguistic Research 27.2: 285-319.

" Foley, William A. y Robert D. Van Valin Jr. 1984. Functional syntax and universal grammar. Cambridge Studies in Linguistics 38. Cambridge: Cambridge University Press.

» Frazier, Lyn y Janet Dean Fodor. 1978. "The sausage machine: A new two-stage parsing model". Cognition 6.4: 291-325.

" Friederici, Angela D. 1998. "The neurobiology of language comprehension”. En Language comprehension: A biological perspective, editado por Angela D. Friederici, 263-301. Berlin: Springer.

"Friederici, Angela D. 2002. "Towards a neural basis of auditory sentence processing". Trends in Cognitive Sciences 6.2: 78-84.

»Friederici, Angela D. 2004. "Processing local transitions versus long-distance syntactic hierarchies". Trends in Cognitive Sciences 8.6: 245-247.

» Friederici, Angela D., Karsten Steinhauer, Axel Mecklinger y Martin Meyer. 1998. “Working memory constraints on syntactic ambiguity resolution as revealed by electrical brain responses". Biological Psychology 47.3: 193-221.

" Frisch, Stefan y Matthias Schlesewsky. 2001. "The N400 reflects problems of thematic hierarchizing". NeuroReport 12.15: 3391-3394.

» Frisch, Stefan, Matthias Schlesewsky y H. Wegner. 2005. "The interaction of morphological case and word order constraints: Cross-linguistic ERP evidence from German, Russian and Finnish". Conference paper. 12th Annual Meeting of the Cognitive-Neuroscience-Society.

" Gattei, Carolina A., Michael W. Dickey, Alejandro J. Wainselboim y Luis París. 2015. “The thematic hierarchy in sentence comprehension: A study on the interaction between verb class and word order in Spanish". The Quarterly Journal of Experimental Psychology 68.10: 1981-2007.

" Gattei, Carolina A., Yamila Sevilla, Ángel J. Tabullo, Alejandro J. Wainselboim, Luis A. París y Diego E. Shalom. 2018. "Prominence in Spanish sentence comprehension: an eye-tracking study". Language, Cognition and Neuroscience 33.5: 587-607.

» Gattei, Carolina A., Ángel Tabullo, Luis París y Alejandro J. Wainselboim. 2015. "The role of prominence in Spanish sentence comprehension: An ERP study". Brain and Language 150: $22-35$.

" Gibson, Edward. 1998. “Linguistic complexity: Locality of syntactic dependencies”. Cognition 68 (1):1-76. 
" Gibson, Edward. 200o. "The dependency locality theory: A distance-based theory of linguistic complexity". Image, Language, Brain: Papers from the First Mind Articulation Project Symposium, 95-126.

» Gorrell, Paul. 1995. Syntax and parsing. Cambridge Studies in Linguistics 76. Cambridge, MA: Cambridge University Press.

» Gunter, Thomas C., Laurie A. Stowe, y Gusbertus Mulder. 1997. "When syntax meets semantics". Psychophysiology 34.6: 660-676.

» Hagoort, Peter, Colin M. Brown y Jolanda Groothusen. 1993. “The syntactic positive shift (SPS) as an ERP measure of syntactic processing”. Language and Cognitive Processes 8.4: 439-483.

» Hagoort, Peter y Colin M. Brown. 200o. “ERP effects of listening to speech compared to reading: the P6oo/SPS to syntactic violations in spoken sentences and rapid serial visual presentation". Neuropsychologia 38.11: 1531-1549.

» Hagoort, Peter, Lea Hald, Marcel Bastiaansen y Karl Magnus Petersson. 2004. “Integration of word meaning and world knowledge in language comprehension". Science 304.5669:438-441.

»Hahne, Anja. 1997. Charakteristika syntaktischer und semantischer Prozesse bei der auditiven Sprachverarbeitung: Evidenz aus ereigniskorrelierten Potentialstudien. Leipzig: Max Planck Institute of Cognitive Neuroscience.

» Hahne, Anja y Angela D. Friederici. 1999. "Electrophysiological evidence for two steps in syntactic analysis: Early automatic and late controlled processes". Journal of Cognitive Neuroscience 11.2: 194-205.

»Inoue, Atsu y Janet Dean Fodor. 1995. “Information-paced parsing of Japanese". Japanese sentence processing, editado por Reiko Mazuka y Noriko Nagai, 9-63. Hillsdale, NJ: Lawrence Erlbaum.

»Kaan, Edith, Anthony Harris, Edward Gibson y Phillip Holcomb. 2000. "The P6oo as an index of syntactic integration difficulty”. Language and Cognitive Processes 15.2: 159-201.

"Kaan, Edith y Tamara Swaab. 2003. "Repair, revision, and complexity in syntactic analysis: An electrophysiological differentiation”. Journal of Cognitive Neuroscience 15.1: 98-110.

» Kamide, Yuki. 2008. “Anticipatory processes in sentence processing”. Language and Linguistics Compass 2.4: 647-670.

" Kamide, Yuki y Don C. Mitchell. 1999. "Incremental pre-head attachment in Japanese parsing”. Language and Cognitive Processes 14.5-6: 631-662.

» Kempen, Gerard y Karin Harbusch. 2005. "The relationship between grammaticality ratings and corpus frequencies: A case study into word order variability in the midfield of German clauses". Linguistic Evidence: Empirical, Theoretical, and Computational Perspectives 85: 329.

» Kim, Albert y Lee Osterhout. 2005. "The independence of combinatory semantic processing: Evidence from event-related potentials”. Journal of Memory and Language 52.2: 205-225.

» Kimball, John. 1975. "Predictive analysis and over-the-top parsing”. Syntax and Semantics 4: 155-179.

» Konieczny, Lars. 2000. “Locality and parsing complexity”. Journal of Psycholinguistic Research 29.6: 627-645.

» Kosta, Peter. 2006. "On free word order phenomena in Czech as compared to German: Is clause internal scrambling A-movement, A-bar-movement or is it base generated?”. Zeitschrift für Slawistik 51.3: 306-320. 
» Kutas, Marta, Katherine A. DeLong y Nathaniel J. Smith. 2011. "A look around at what lies ahead: Prediction and predictability in language processing". Predictions in the brain: Using our past to generate a future, editado por Moshe Bar, 190-207. Oxford: Oxford University Press.

» Kutas, Marta y Steven A Hillyard. 1983. “Event-related brain potentials to grammatical errors and semantic anomalies”. Memory \& Cognition 11.5: 539-550.

»Lamers, Monica J. 2001. Sentence processing: Using syntactic, semantic, and thematic information. Groningen: University of Groningen.

» Lewis, Richard L. y Shravan Vasishth. 2005. “An activation-based model of sentence processing as skilled memory retrieval”. Cognitive Science 29.3: 375-419.

» MacWhinney, Brian y Elizabeth Bates, eds. 1989. The crosslinguistic study of sentence processing. Problems in the Behavioural Sciences. Cambridge: Cambridge University Press.

» MacWhinney, Brian, Elizabeth Bates y Reinhold Kliegl. 1984. "Cue validity and sentence interpretation in English, German, and Italian". Journal of Verbal Learning and Verbal Behavior 23.2: 127-150.

» McRae, Ken, Todd R. Ferretti y Liane Amyote. 1997. "Thematic roles as verb-specific concepts”. Language and Cognitive Processes 12.2-3: 137-176.

» Metzner, Paul, Titus von der Malsburg, Shravan Vasishth y Frank Rösler. 2015. “Brain responses to world knowledge violations: A comparison of stimulus-and fixation-triggered eventrelated potentials and neural oscillations". Journal of Cognitive Neuroscience 27.5: 1017-1028.

» Neville, Helen, Janet L. Nicol, Andrew Barss, Kenneth I. Forster y Merrill F. Garrett. 1991. "Syntactically based sentence processing classes: Evidence from event-related brain potentials". Journal of Cognitive Neuroscience 3.2: 151-165.

» Osterhout, Lee y Phillip J. Holcomb. 1992. "Event-related brain potentials elicited by syntactic anomaly”. Journal of Memory and Language 31.6: 785-806.

»Osterhout, Lee y Phillip J. Holcomb. 1993. "Event-related potentials and syntactic anomaly: Evidence of anomaly detection during the perception of continuous speech". Language and Cognitive Processes 8.4: 413-437.

»Osterhout, Lee y Linda A. Mobley. 1995. “Event-related brain potentials elicited by failure to agree”. Journal of Memory and Language 34.6: 739-773.

» Roehm, Dietmar. 2004. “Waves and words: Oscillatory activity and language processing”. Tesis de doctorado, Philipps-Universität Marburg.

» Roehm, Dietmar, Ina Bornkessel, Hubert Haider y Matthias Schlesewsky. 2005. “When case meets agreement: Event-related potential effects for morphology-based conflict resolution in human language comprehension". NeuroReport 16.8: 875-878.

» Roehm, Dietmar, Matthias Schlesewsky, Ina Bornkessel, Stefan Frisch y Hubert Haider. 2004. "Fractionating language comprehension via frequency characteristics of the human EEG”. NeuroReport 15.3: 409-412.

»Steinhauer, Karsten, Axel Mecklinger, Angela D. Friederici y Martin Meyer. 1997. "Wahrscheinlichkeit und Strategie: Eine EKP-Studie zur Verarbeitung syntaktischer Anomalien”. Zeitschrift für Experimentelle Psychologie 19.2: 305-331.

» Tanenhaus, Michael K., Michael J. Spivey-Knowlton, Kathleen M. Eberhard y Julie C. Sedivy. 1995. "Integration of visual and linguistic information in spoken language comprehension”. Science 268.5217: 1632-1634.

»Van Berkum, Jos J. A., Colin M. Brown, Pienie Zwitserlood, Valesca Kooijman y Peter Hagoort. 2005. "Anticipating upcoming words in discourse: evidence from ERPs and reading times”. Journal of Experimental Psychology: Learning, Memory, and Cognition 31.3: 443. 
"Van Valin Jr., Robert D. 2005. Exploring the syntax-semantics interface. Cambridge: Cambridge University Press.

»Van Valin, Robert D. y Randy J. LaPolla. 1997. Syntax: Structure, meaning, and function: Cambridge University Press.

"Wang, Luming, Matthias Schlesewsky, Balthasar Bickel e Ina Bornkessel-Schlesewsky. 2009. "Exploring the nature of the 'subject'-preference: Evidence from the online comprehension of simple sentences in Mandarin Chinese". Language and Cognitive Processes 24.7-8: 1180-1226.

"Wang, Luming, Matthias Schlesewsky, Balthasar Bickel e Ina Bornkessel-Schlesewsky. 2012. "The role of animacy in online argument interpretation in Mandarin Chinese". Case, word order and prominence: Interacting cues in language production and comprehension, editado por Monique Lamers y Peter de Swart, 91-119. Studies in Theoretical Psycholinguistics. Dordrecht: Springer.

"Weckerly, jill y Marta Kutas. 1999. "An electrophysiological analysis of animacy effects in the processing of object relative sentences". Psychophysiology 36.5: 559-570.

»Wicha, Nicole Y. Y., Eva M. Moreno y Marta Kutas. 2004. "Anticipating words and their gender: An event-related brain potential study of semantic integration, gender expectancy, and gender agreement in Spanish sentence reading". Journal of Cognitive Neuroscience 16.7: 1272-1288. 